\title{
AN ANALOGUE OF THE ERDÖS-GINZBURG-ZIV THEOREM FOR QUADRATIC SYMMETRIC POLYNOMIALS
}

\author{
Arie Bialostocki \\ Department of Mathematics, University of Idaho, Moscow ID 83844 USA \\ arieb@uidaho.edu \\ Tran Dinh Luong ${ }^{1}$ \\ Department of Mathematics, University of Idaho, Moscow ID 83844 USA \\ luongtran@vandals. uidaho.edu
}

Received: 12/23/08, Accepted: 5/20/09, Publsihed: 9/25/09

\begin{abstract}
Let $p$ be a prime and let $\varphi \in \mathbb{Z}_{p}\left[x_{1}, x_{2}, \ldots, x_{p}\right]$ be a symmetric polynomial, where $\mathbb{Z}_{p}$ is the field of $p$ elements. A sequence $T$ in $\mathbb{Z}_{p}$ of length $p$ is called a $\varphi$-zero sequence if $\varphi(T)=0$; a sequence in $\mathbb{Z}_{p}$ is called a $\varphi$-zero free sequence if it does not contain any $\varphi$-zero subsequence. Define $g\left(\varphi, \mathbb{Z}_{p}\right)$ to be the smallest integer $l$ such that every sequence in $\mathbb{Z}_{p}$ of length $l$ contains a $\varphi$-zero sequence; if $l$ does not exist, we set $g\left(\varphi, \mathbb{Z}_{p}\right)=\infty$. Define $M\left(\varphi, \mathbb{Z}_{p}\right)$ to be the set of all $\varphi$-zero free sequences of length $g\left(\varphi, \mathbb{Z}_{p}\right)-1$, whenever $g\left(\varphi, \mathbb{Z}_{p}\right)$ is finite. The aim of this paper is to determine the value of $g\left(\varphi, \mathbb{Z}_{p}\right)$ and to describe the set $M\left(\varphi, \mathbb{Z}_{p}\right)$ for a quadratic symmetric polynomial $\varphi$ in $\mathbb{Z}_{p}\left[x_{1}, x_{2}, \ldots, x_{p}\right]$.
\end{abstract}

\section{Introduction}

This paper is motivated by the following theorem of Erdös, Ginzburg, and Ziv, [9], stated below in Theorem 1.1 (i) for a prime. Part (ii) of Theorem 1.1 addresses the inverse problem which corresponds to the first part. Several new proofs of (i) appear in [1], and a proof of (ii) appears in [16] and [5]; see also [15].

Theorem 1 (EGZ) Let $p$ be a prime and let $\mathbb{Z}_{p}$ be the additive group of residue classes modulo $p$.

(i) Every sequence in $\mathbb{Z}_{p}$ of length $2 p-1$ contains a zero-sum subsequence of length $p$.

(ii) The set of all sequences of maximal length in $\mathbb{Z}_{p}$ that do not contain any zerosum subsequence of length $p$ is that of all sequences containing exactly two distinct elements, where each element appears $p-1$ times.

There were numerous generalizations and developments of the EGZ theorem in recent years; a comprehensive list of references on this topic can be found in the surveys [8], [2], [3], [10] and [11]. This paper diverts from most previous works, as it takes into consideration the field structure of $\mathbb{Z}_{p}$ rather than being restricted to its additive structure. More precisely, we deal with symmetric polynomials in $p$ variables

\footnotetext{
${ }^{1}$ Partially supported by Project 322, Ministry of Education of Vietnam.

This paper is part of the second author's dissertation under the supervision of the first author.
} 
over $\mathbb{Z}_{p}$, motivated by the fact that the sum in the EGZ theorem corresponds to the first elementary symmetric polynomial in $\mathbb{Z}_{p}\left[x_{1}, x_{2}, \ldots, x_{p}\right]$. It is worthwhile to mention some historical origins to our approach. Two zero-sum problems concerning the ring $\mathbb{Z}_{n}$ were raised in [4, p. 125], and independently the weighted version of the EGZ theorem, [13], was conjectured in [8, p. 96].

We start by introducing some definitions and notations. Let $p$ be a prime and let $\mathbb{Z}_{p}$ be the prime field of $p$ elements. Let $\varphi$ be a symmetric polynomial in $\mathbb{Z}_{p}\left[x_{1}, x_{2}, \ldots, x_{p}\right]$. A sequence of $p$ elements $a_{1}, a_{2}, \ldots, a_{p}$ in $\mathbb{Z}_{p}$ is called a $\varphi$-zero sequence if $\varphi\left(a_{1}, a_{2}, \ldots, a_{p}\right)=0$; a sequence in $\mathbb{Z}_{p}$ is called $\varphi$-zero free if it does not contain any $\varphi$-zero subsequence. Define $g\left(\varphi, \mathbb{Z}_{p}\right)$ to be the smallest integer $l$ such that every sequence in $\mathbb{Z}_{p}$ of length $l$ contains a $\varphi$-zero subsequence; if $l$ does not exist, we set $g\left(\varphi, \mathbb{Z}_{p}\right)=\infty$. Define $M\left(\varphi, \mathbb{Z}_{p}\right)$ to be the set of all $\varphi$-zero free sequences of length $g\left(\varphi, \mathbb{Z}_{p}\right)-1$, whenever $g\left(\varphi, \mathbb{Z}_{p}\right)$ is finite. We consider two sequences in $\mathbb{Z}_{p}$ to be identical if they differ by the order of their elements, and use the notation $\left[a_{1}\right]^{\alpha_{1}}\left[a_{2}\right]^{\alpha_{2}} \ldots\left[a_{k}\right]^{\alpha_{k}}$ to denote a sequence in $\mathbb{Z}_{p}$ where each element $a_{i}$ appears $\alpha_{i}$ times.

Let $\varphi$ be a symmetric polynomial in $\mathbb{Z}_{p}\left[x_{1}, x_{2}, \ldots, x_{p}\right]$. It is clear that if we have $\varphi(0,0, \ldots, 0) \neq 0$, then for every integer $m$, where $m \geqslant 1$, the sequence $[0]^{m}$ is $\varphi$-zero free, which implies $g\left(\varphi, \mathbb{Z}_{p}\right)=\infty$. We now suppose $\varphi(0,0, \ldots, 0)=0$. If $\varphi$ is a linear symmetric polynomial, then, by the EGZ theorem, we have $g\left(\varphi, \mathbb{Z}_{p}\right)=2 p-1$ and $M\left(\varphi, \mathbb{Z}_{p}\right)$ is the set of all sequences in $\mathbb{Z}_{p}$ of the form $[u]^{p-1}[v]^{p-1}$, where $u, v \in \mathbb{Z}_{p}$ and $u \neq v$. In this paper, we will determine the value of $g\left(\varphi, \mathbb{Z}_{p}\right)$ and describe the set $M\left(\varphi, \mathbb{Z}_{p}\right)$ for a quadratic symmetric polynomial $\varphi$ in $\mathbb{Z}_{p}\left[x_{1}, x_{2}, \ldots, x_{p}\right]$.

Throughout the paper we will denote by $d(T)$ the number of distinct elements of a sequence $T$ in $\mathbb{Z}_{p}$, and denote by $s_{k}$, for $k \geqslant 1$, the power-sum symmetric polynomial of degree $k$ in $\mathbb{Z}_{p}\left[x_{1}, x_{2}, \ldots, x_{p}\right]$, which is defined by the formula $s_{k}\left(x_{1}, x_{2}, \ldots, x_{p}\right)=$ $x_{1}^{k}+x_{2}^{k}+\cdots+x_{p}^{k}$.

\section{Main Result}

Let $p$ be a prime, where $p \geqslant 3$, and let $\varphi \in \mathbb{Z}_{p}\left[x_{1}, x_{2}, \ldots, x_{p}\right]$ be a quadratic symmetric polynomial with $\varphi(0,0, \ldots, 0)=0$. Then $\varphi$ can be written in the form $a s_{1}^{2}+b s_{2}+c s_{1}$, where $a, b, c \in \mathbb{Z}_{p}$, and either $a \neq 0$ or $b \neq 0$.

The main result of the paper is the following theorem.

Theorem 2 Let $p$ be a prime, where $p \geqslant 3$, and let $\varphi=a s_{1}^{2}+b s_{2}+c s_{1}$, where $a, b, c \in \mathbb{Z}_{p}$, and either $a \neq 0$ or $b \neq 0$, be a quadratic symmetric polynomial in $\mathbb{Z}_{p}\left[x_{1}, x_{2}, \ldots, x_{p}\right]$. Then the following assertions hold:

(i) If $a=0$ and $b \neq 0$, then $g\left(\varphi, \mathbb{Z}_{p}\right)=2 p-1$, and $M\left(\varphi, \mathbb{Z}_{p}\right)$ is the set of all sequences of the form

$$
[u]^{\alpha}\left[-u-c b^{-1}\right]^{p-1-\alpha}[v]^{\beta}\left[-v-c b^{-1}\right]^{p-1-\beta},
$$

where $u, v \in \mathbb{Z}_{p}, u \neq v, u+v \neq-c b^{-1}$ and $0 \leqslant \alpha \leqslant p-1,0 \leqslant \beta \leqslant p-1$. 
(ii) If $a \neq 0, b=0$ and $c=0$, then $g\left(\varphi, \mathbb{Z}_{p}\right)=2 p-1$, and $M\left(\varphi, \mathbb{Z}_{p}\right)$ is the set of all sequences of the form $[u]^{p-1}[v]^{p-1}$, where $u, v \in \mathbb{Z}_{p}$ and $u \neq v$.

(iii) If $a \neq 0, b=0$ and $c \neq 0$, then $g\left(\varphi, \mathbb{Z}_{p}\right)=2 p-2$, and $M\left(\varphi, \mathbb{Z}_{p}\right)$ is the set of all sequences of the form $[u]^{p-1}\left[u+c a^{-1}\right]^{p-2}$, where $u \in \mathbb{Z}_{p}$.

(iv) If $a \neq 0, b \neq 0$ and $p \geqslant 5$, then

$$
2(p-1)+n(p) \leqslant g\left(\varphi, \mathbb{Z}_{p}\right) \leqslant 4 p-3,
$$

where $n(p)$ denotes the least quadratic non-residue modulo $p$.

The following two results will be used in the proof of Theorem 2.1.

Lemma 3 Let $m \geqslant 4$, and let $S$ be a sequence in $\mathbb{Z}_{m}$ of length $2 m-3$.

(i) ([7]) If $S$ has at least four distinct elements, then it contains a zero-sum subsequence of length $m$.

(ii) $([6,11])$ If $S$ does not contain any zero-sum subsequence of length $m$, then it either has the form $[u]^{m-1}[v]^{m-2}$ or $[u]^{m-1}[v]^{m-3}[2 v-u]^{1}$, where $u, v \in \mathbb{Z}_{m}$, and $\operatorname{gcd}(u-v, m)=1$.

Lemma $4([12,14])$ Every sequence in $\mathbb{Z}_{m} \oplus \mathbb{Z}_{m}$ of length $4 m-3$ contains a zero-sum subsequence of length $\mathrm{m}$.

Proof of Theorem 2. (i) Suppose $a=0$ and $b \neq 0$. Then we have $\varphi=b s_{2}+c s_{1}$. Let $f(x)=b x^{2}+c x \in \mathbb{Z}_{p}[x]$. Then

$$
\varphi\left(x_{1}, x_{2}, \ldots, x_{p}\right)=f\left(x_{1}\right)+f\left(x_{2}\right)+\cdots+f\left(x_{p}\right) .
$$

Let $a_{1}, a_{2}, \ldots, a_{2 p-1}$ be a sequence in $\mathbb{Z}_{p}$ of length $2 p-1$. Then, by the EGZ theorem, the sequence $f\left(a_{1}\right), f\left(a_{2}\right), \ldots, f\left(a_{2 p-1}\right)$ contains a zero-sum subsequence of length $p$. It follows that the former sequence contains a $\varphi$-zero subsequence, which implies $g\left(\varphi, \mathbb{Z}_{p}\right) \leqslant 2 p-1$.

Next let $b_{1}, b_{2}, \ldots, b_{2 p-2}$ be a sequence in $\mathbb{Z}_{p}$ of length $2 p-2$. It is clear that this sequence is $\varphi$-zero free if and only if the sequence $f\left(b_{1}\right), f\left(b_{2}\right), \ldots, f\left(b_{2 p-2}\right)$ does not contain any zero-sum subsequence of length $p$. By the EGZ theorem, this is equivalent to the fact that the later sequence is of the form $[y]^{p-1}[z]^{p-1}$, where $y, z \in \mathbb{Z}_{p}$ and $y \neq z$. Since the value set of $f(x)$ for $x \in \mathbb{Z}_{p}$ contains at least two distinct elements, it follows that there exists a $\varphi$-zero free sequence in $\mathbb{Z}_{p}$ of length $2 p-2$. Hence $g\left(\varphi, \mathbb{Z}_{p}\right)=2 p-1$. Furthermore, a simple computation shows that for $u, v \in \mathbb{Z}_{p}, u \neq v$, the equality $f(u)=f(v)$ holds if and only if $u+v=-c b^{-1}$. Therefore $M\left(\varphi, \mathbb{Z}_{p}\right)$ is the set of all sequences of the form in (i).

(ii) Suppose $a \neq 0, b=0$ and $c=0$. Then $\varphi=a s_{1}^{2}$, and (ii) follows by the EGZ theorem. 
(iii) Suppose $a \neq 0, b=0$ and $c \neq 0$. Without loss of generality, we may assume that $a=1$. Then we have $\varphi=s_{1}^{2}+c s_{1}$.

Let $S$ be a sequence in $\mathbb{Z}_{p}$ of length $2 p-2$. We will show that $S$ contains a $\varphi$-zero subsequence, which implies $g\left(\varphi, \mathbb{Z}_{p}\right) \leqslant 2 p-2$. The case $d(S)=1$ is trivial; the case $d(S) \geqslant 3$ follows by the EGZ theorem (ii). We now consider the case $d(S)=2$. If $S$ has an element appearing more than $p-1$ times, then it is clear that $S$ contains a zero-sum subsequence of length $p$, which is also a $\varphi$-zero subsequence. So we may asssume that $S=[u]^{p-1}[v]^{p-1}$, where $u, v \in \mathbb{Z}_{p}$ and $u \neq v$. Let $\alpha$ be the integer such that $0 \leqslant \alpha \leqslant p-1$ and $\alpha \equiv c(v-u)^{-1}(\bmod p)$, and let $T=[u]^{\alpha}[v]^{p-\alpha}$. It is clear that $\alpha \neq 0$, and hence $T$ is a subsequence of $S$ of length $p$. A simple computation shows that $s_{1}(T)=\alpha(u-v)=-c$. It follows that $\varphi(T)=0$, and hence $T$ is a $\varphi$-zero subsequence of $S$.

Let $V=[u]^{p-1}[u+c]^{p-2}$, where $u \in \mathbb{Z}_{p}$. If $T$ is a subsequence of $V$ of length $p$, then it has the form $[u]^{p-\alpha}[u+c]^{\alpha}$, where $1 \leqslant \alpha \leqslant p-2$. A simple computation shows that

$$
\varphi(T)=\alpha c(\alpha c+c)=c^{2} \alpha(\alpha+1) \neq 0,
$$

and hence $V$ is $\varphi$-zero free. Thus we have proved that $g\left(\varphi, \mathbb{Z}_{p}\right)=2 p-2$.

We now describe the set $M\left(\varphi, \mathbb{Z}_{p}\right)$. The argument above shows that all the sequences of the form $[u]^{p-1}[u+c]^{p-2}$, where $u \in \mathbb{Z}_{p}$, belong to $M\left(\varphi, \mathbb{Z}_{p}\right)$. Now let $U$ be a $\varphi$-zero free sequence in $\mathbb{Z}_{p}$ of length $2 p-3$. It is clear that if $d(U)=1$, then $U$ contains a $\varphi$-zero sequence, a contradiction. If $d(U) \geqslant 4$, then, by Lemma 2.2 (i), it contains a zero-sum subsequence of length $p$, which is also a $\varphi$-zero sequence, a contradiction.

We claim that $d(U) \neq 3$. Suppose, to the contrary, that $d(U)=3$. If $p=3$, then $U=[0]^{1}[1]^{1}[2]^{1}$, and it is clear that $U$ is a $\varphi$-zero sequence, a contradiction. So we may assume $p \geqslant 5$. Since $U$ is $\varphi$-zero free, it follows that $U$ does not contain any zero-sum subsequence of length $p$. Hence, by Lemma 2.2 (ii), it must be of the form

$$
U=[u]^{p-1}[u+w]^{p-3}[u+2 w]^{1},
$$

where $u, w \in \mathbb{Z}_{p}$ and $w \neq 0$. We consider three cases of $w$.

Case $1: w \neq c$ and $w \neq c 2^{-1}$. Let $\alpha$ be the integer such that $0 \leqslant \alpha \leqslant p-1$ and $\alpha \equiv c w^{-1}(\bmod p)$, and let $T=[u]^{\alpha}[u+w]^{p-\alpha}$. It is clear that $\alpha \notin\{0,1,2\}$, and hence $T$ is a subsequence of $U$ of length $p$. A simple computation shows that $\varphi(T)=-\alpha w(-\alpha w+c)=0$. Hence $T$ is a $\varphi$-zero subsequence of $U$, a contradiction.

Case $2: w=c$. Let $T=[u]^{2}[u+w]^{p-3}[u+2 w]^{1}$. A simple computation shows that $\varphi(T)=-w(-w+c)=0$. Hence $T$ is a $\varphi$-zero subsequence of $U$, a contradiction.

Case $3: w=c 2^{-1}$. Let $T=[u]^{3}[u+w]^{p-4}[u+2 w]^{1}$. A simple computation shows that $\varphi(T)=-2 w(-2 w+c)=0$. Hence $T$ is a $\varphi$-zero subsequence of $U$, a contradiction. 
Thus we have proved that $d(U) \neq 3$, and our claim follows. Hence we must have $d(U)=2$. Since $U$ is a $\varphi$-zero free sequence, it does not contain any zero-sum subsequence of length $p$. Hence, by Lemma 2.2 (ii) again, it must be of the form

$$
U=[u]^{p-1}[v]^{p-2},
$$

where $u, v \in \mathbb{Z}_{p}$ and $u \neq v$. We will show that $v-u=c$. Suppose, to the contrary, that $v-u \neq c$. Let $\alpha$ be the integer such that $0 \leqslant \alpha \leqslant p-1$ and $\alpha \equiv c(v-u)^{-1}$ $(\bmod p)$, and let $T=[u]^{\alpha}[v]^{p-\alpha}$. It is clear that $\alpha \notin\{0,1\}$, and hence $T$ is a subsequence of $U$ of length $p$. A simple computation shows that

$$
\varphi(T)=\alpha(u-v)(\alpha(u-v)+c)=0
$$

Hence $T$ is a $\varphi$-zero subsequence of $U$, a contradiction. Thus we have $v-u=c$, and (iii) follows.

(iv) Suppose $a \neq 0, b \neq 0$ and $p \geqslant 5$. Without loss of generality, we may assume that $a=1$. Then we have $\varphi=s_{1}^{2}+b s_{2}+c s_{1}$. By the change of variables $x_{i} \mapsto$ $x_{i}-c(2 b)^{-1}$ for $1 \leqslant i \leqslant p$, the polynomial $\varphi$ becomes $s_{1}^{2}+b s_{2}$. So, without loss of generality, we may also assume that $c=0$. We first prove that $g\left(\varphi, \mathbb{Z}_{p}\right) \leqslant 4 p-3$. Let $a_{1}, a_{2}, \ldots, a_{4 p-3}$ be a sequence in $\mathbb{Z}_{p}$ of length $4 p-3$. By Lemma 2.3 , the sequence $\left(a_{1}, a_{1}^{2}\right),\left(a_{2}, a_{2}^{2}\right), \ldots,\left(a_{4 p-3}, a_{4 p-3}^{2}\right)$ in $\mathbb{Z}_{p} \oplus \mathbb{Z}_{p}$ contains a zero-sum subsequence of length $p$. It follows that the former sequence contains a subsequence, say $T$, that is an $s_{1}$-zero and $s_{2}$-zero sequence simultaneously. It is clear that $T$ is also a $\varphi$-zero sequence, and hence the required inequality follows.

We now establish the lower bound for $g\left(\varphi, \mathbb{Z}_{p}\right)$.

Claim There exists $u \in \mathbb{Z}_{p}$ such that $b\left(1-u^{2}\right)$ is a quadratic non-residue in $\mathbb{Z}_{p}$.

Let $A$ be the set of all squares in $\mathbb{Z}_{p}$ and let $B=\{b(1-x) \mid x \in A\}$. Since $p \geqslant 5$, there exists a quadratic non-residue $w$ in $\mathbb{Z}_{p}$ with $w \neq-1$. Then we have $\sum_{x \in A} x+w \sum_{x \in A} x=\sum_{y \in \mathbb{Z}_{p}} y=0$. Since $w \neq-1$, it follows that

$$
\sum_{x \in A} x=0
$$

Hence,

$$
\sum_{y \in B} y=\sum_{x \in A} b(1-x)=b \sum_{x \in A} 1-b \sum_{x \in A} x=b(p+1) / 2
$$

If $A=B$, then $\sum_{y \in B} y=\sum_{x \in A} x=0$, which implies $b(p+1) / 2=0$, a contradiction. Hence $A \neq B$. Since $|A|=|B|$, it follows that there exists an element $u \in \mathbb{Z}_{p}$ such that $b\left(1-u^{2}\right) \notin A$, and our claim follows.

Let us consider the sequence

$$
U=[1]^{p-1}[-1]^{p-1}[u]^{n(p)-1}
$$


where $u$ is chosen so that $b\left(1-u^{2}\right)$ is a quadratic non-residue in $\mathbb{Z}_{p}$, and $n(p)$ denotes the least quadratic non-residue modulo $p$. To prove $g\left(\varphi, \mathbb{Z}_{p}\right) \geqslant 2(p-1)+n(p)$, we show that the sequence $U$ is $\varphi$-zero free. Suppose, to the contrary, that $U$ contains a $\varphi$-zero subsequence of length $p$, say

$$
T=[1]^{\alpha}[-1]^{\beta}[u]^{\gamma},
$$

where $0 \leqslant \alpha, \beta \leqslant p-1,0 \leqslant \gamma \leqslant n(p)-1$ and $\alpha+\beta+\gamma=p$. A simple computation shows that $\varphi(T)=0$ implies the equality

$$
(\alpha-\beta+u \gamma)^{2}-b\left(1-u^{2}\right) \gamma=0 .
$$

If $\gamma=0$, then it follows that $\alpha-\beta \equiv 0(\bmod p)$, which is impossible since $0 \leqslant$ $\alpha, \beta \leqslant p-1$ and $\alpha+\beta=p$. If $1 \leqslant \gamma \leqslant n(p)-1$, then $\gamma$ is a quadratic residue in $\mathbb{Z}_{p}$. Hence $b\left(1-u^{2}\right) \gamma$ is a quadratic non-residue in $\mathbb{Z}_{p}$, a contradiction. Thus we have proved that the sequence $U$ is $\varphi$-zero free, and (iv) follows.

The proof of the theorem is complete.

Remark Let us consider the case that $\varphi=a s_{1}^{2}+b s_{2}+c s_{1}$, where $a, b, c \in \mathbb{Z}_{p}$, $a \neq 0$ and $b \neq 0$, as in Theorem 2.1 (iv). We note that the inequality $g\left(\varphi, \mathbb{Z}_{p}\right) \geqslant$ $2(p-1)+n(p)$ does not hold for $p=3$. Indeed, a direct computation shows that $g\left(\varphi, \mathbb{Z}_{3}\right)=5$ if $b=a$, and $g\left(\varphi, \mathbb{Z}_{3}\right)=6$ if $b=-a$, while $2(p-1)+n(p)=6$ if $p=3$.

The problem of finding the value of $g\left(\varphi, \mathbb{Z}_{p}\right)$, where $\varphi$ has the form above, for $p \geqslant 5$ is still open. A computer aided computation shows that $g\left(\varphi, \mathbb{Z}_{5}\right)=11$ if $b=a$, and $g\left(\varphi, \mathbb{Z}_{5}\right)=10$ if $b \neq a$; and $g\left(\varphi, \mathbb{Z}_{7}\right)=17$ if $b=-2 a$, and $g\left(\varphi, \mathbb{Z}_{7}\right)=15$ if $b \neq-2 a$. It can be seen that the lower bound for $g\left(\varphi, \mathbb{Z}_{p}\right)$ in Theorem 2.1 (iv) is sharp for $p=5,7$.

Acknowledgements. The authors would like to thank the anonymous referee for his valuable comments and suggestions.

\section{References}

[1] N. Alon and M. Dubiner, Zero-sum sets of prescribed size, Combinatorics, Paul Erdős is eighty, Vol. 1, Bolyai Soc. Math. Stud., János Bolyai Math. Soc., (Budapest, 1993), 33-50.

[2] A. Bialostocki, Zero sum trees: a survey of results and open problems, Finite and infinite combinatorics in sets and logic (Banff, AB, 1991), 19-29, NATO Adv. Sci. Inst. Ser. C Math. Phys. Sci., 411, Kluwer Acad. Publ., Dordrecht, 1993.

[3] A. Bialostocki, Some problems in view of recent developments of the Erdös-Ginzburg-Ziv theorem, Combinatorial number theory, 111-120, de Gruyter, Berlin, 2007. 
[4] A. Bialostocki and P. Dierker, Zero sum Ramsey theorems, Proceedings of the Twentieth Southeastern Conference on Combinatorics, Graph Theory, and Computing (Boca Raton, FL, 1989). Congr. Numer. 70 (1990) 119-130.

[5] A. Bialostocki and P. Dierker, On the Erdös-Ginzburg-Ziv theorem and the Ramsey numbers for stars and matchings, Discrete Math. 110 (1992), no. 1-3, 1-8.

[6] A. Bialostocki, P. Dierker, D. Grynkiewicz, and M. Lotspeich, On some developments of the Erdös-Ginzburg-Ziv theorem II, Acta Arith. 110 (2003), no. 2, 173-184.

[7] A. Bialostocki and M. Lotspeich, Some developments of the Erdös-Ginzburg-Ziv theorem I, Sets, graphs and numbers (Budapest, 1991), 97-117, Colloq. Math. Soc. János Bolyai 60, North-Holland, Amsterdam, 1992.

[8] Y. Caro, Zero-sum problems-a survey, Discrete Math. 152 (1996), no. 1-3, 93-113.

[9] P. Erdős, A. Ginzburg, and A. Ziv, Theorem in additive number theory, Bull. Res. Council Israel 10F (1961), 41-43.

[10] W. Gao and A. Geroldinger, Zero-sum problems in finite abelian groups: a survey, Expo. Math. 24 (2006) no. 4, 337-369.

[11] A. Geroldinger, Additive group theory and non-unique factorizations, Combinatorial Number Theory and Additive Group Theory (A. Geroldinger and I. Ruzsa, eds.), Advanced Courses in Mathematics CRM Barcelona, Birkhäuser, 2009, pp. 1-86.

[12] A. Geroldinger and F. Halter-Koch, Non-Unique Factorizations. Algebraic, Combinatorial and Analytic Theory, Pure and Applied Mathematics 278, Chapman \& Hall/CRC, 2006.

[13] D. J. Grynkiewicz, A weighted Erdös-Ginzburg-Ziv theorem, Combinatorica 26 (2006), no. $4,445-453$.

[14] C. Reiher, On Kemnitz conjecture concerning lattice-points in the plane, Ramanujan J. 13 (2007), no. 1-3, 333-337.

[15] S. Savchev and F. Chen, Long n-zero-free sequences in finite cyclic groups, Discrete Math. 308 (2008), 1-8.

[16] T. Yuster and B. Peterson, A generalization of an addition theorem for solvable groups, Canad. J. Math. 36 (1984) no. 3, 529-536. 